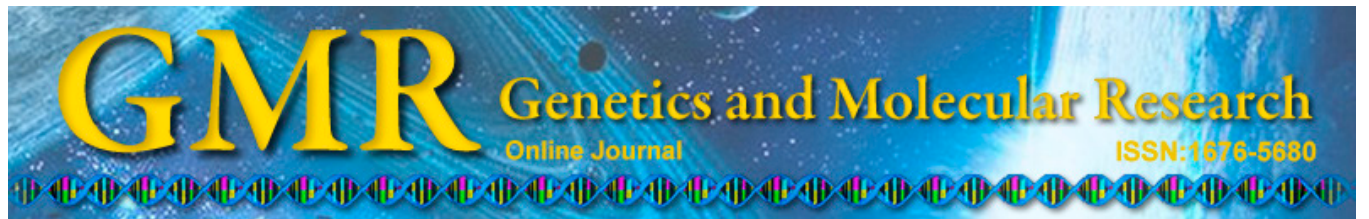

\title{
Effects of hypoxia on proliferation and osteogenic differentiation of periodontal ligament stem cells: an in vitro and in vivo study
}

Q.B. Zhang ${ }^{1 *}$, Z.Q. Zhang ${ }^{2 *}$, S.L. Fang ${ }^{2}$, Y.R. Liu ${ }^{3}$, G. Jiang ${ }^{4}$ and K.F. Li ${ }^{5}$

${ }^{1}$ Key Laboratory of Oral Medicine, Guangzhou Institute of Oral Disease, Stomatology Hospital of Guangzhou Medical University, Guangzhou, Guangdong Province, China

${ }^{2}$ Department of Stomatology,

The Sixth Affiliated Hospital of Sun Yat-Sen University, Guangzhou, Guangdong Province, China

${ }^{3}$ Department of Pediatric Dentistry, Hospital of Stomatology, Guangzhou Medical University, Guangzhou, Guangdong Province, China ${ }^{4}$ Jiangsu Key Laboratory of Biological Cancer Therapy,

Xuzhou Medical College, Xuzhou, China

${ }^{5}$ School of Medicine, University of California, San Diego, CA, USA

*These authors contributed equally to this study.

Corresponding author: Q.B. Zhang

E-mail: doctorqingbin@hotmail.com

Genet. Mol. Res. 13 (4): 10204-10214 (2014)

Received January 8, 2014

Accepted March 24, 2014

Published December 4, 2014

DOI http://dx.doi.org/10.4238/2014.December.4.15

\begin{abstract}
Changes in oxygen concentration may influence various innate characteristics of stem cells. The effects of varying oxygen concentration on human periodontal ligament stem cells (HPDLSCs) has not been explored, particularly under hypoxia-related conditions. First, HPDLSCs were cultured from the periodontium of human teeth using the outgrowth method. STRO-1 and CD146 expression of HPDLSCs was investigated by flow cytometry. To detect the multilineage differentiation capacities of HPDLSCs, osteogenic-like and adipogenic-
\end{abstract}


like states were induced in cells. Next, HPDLSCs (passage 3) were exposed to normal oxygen $\left(21 \% \mathrm{O}_{2}\right)$ or hypoxia $\left(2 \% \mathrm{O}_{2}\right)$ conditions for 7 days and cell proliferation was evaluated. After culture in osteogenic medium for 7 days, osteoblastic differentiation was evaluated by semiquantitative reverse transcription-polymerase chain reaction analysis to detect 3 osteoblastic markers: core-binding factor a 1/runt-related transcription factor 2 , osteocalcin, and osteopontin. In addition, each cell group was incubated with a hydroxyapatite/tricalcium phosphate carrier and transplanted subcutaneously into the back of immunocompromised mice to investigate transplantation differences in vivo. HPDLSCs were isolated, cultured, and successfully identified. After exposure of HPDLSCs to hypoxia for 7 days, the proliferation rate was increased and showed higher osteogenic differentiation potential compared to control cells. After 12 weeks of transplantation, hypoxia-treated HPDLSCs differentiated into osteoblast-like cells that formed bone-like structures. These results suggest that oxygen concentrations affect various aspects of HPDLSC physiology and that hypoxia enhances osteogenic differentiation both in vivo and in vitro. Oxygen concentration may be a critical parameter for HPDLSCs during expansion and differentiation.

Key words: Osteogenic differentiation; Hypoxia; Osteogenesis; Proliferation; Periodontal ligament stem cells

\section{INTRODUCTION}

Periodontal lesions typically lead to the destruction of periodontium components such as the alveolar bone, periodontal ligament, and gingiva (Bruder et al., 1997; Pittenger et al., 1999; Zhu et al., 2005). Jaw cyst, tumor, trauma, and even extraction may also increase the possibility of periodontal loss under specific circumstances (Lombardo et al., 2013). These factors can affect the integrity of tooth attachment and can ultimately result in tooth loss. A major challenge in the field of periodontology has been the development of strategies to predictably regenerate periodontal tissues that are lost as a result of destructive processes (Baksh et al., 2003; Ezashi et al., 2005). Therefore, periodontal regeneration therapy involves the regeneration and restoration of various periodontal components affected by disease or other etiologies to their original form, function, and consistency (Ivanović et al., 2000a). Different methods have been utilized to promote the regenerative process, particularly osteogenic rehabilitation, and the use of barrier membranes for guided tissue regeneration and application of signaling molecules, such as growth factors and enamel matrix proteins, to root surfaces (Ivanović et al., 2000b; Grayson et al., 2006); however, these approaches have shown limited effectiveness. Periodontal ligament cells have been shown to possess stem cell-like properties including self-renewal, clonogenicity, and multi-tissue differentiation potential (Malladi et al., 2006). Seo et al. (2004) first identified that stem cells were present as periodontal ligament cells, which were referred to as periodontal ligament stem cells. Human periodontal ligament stem cells (HPDLSCs) have the ability to self-renew and show multiple differentiation abilities: osteogenic, neural, and adiogenic lineages. In particular, osteogenic differentiation has been investigated for its ability to promote bone regeneration at a large scale. Thus, regulating 
osteogenic differentiation in human periodontal ligament cells has important implications for the development of new therapeutic strategies for treating periodontal defects (Lee and Kemp, 2006). However, the understanding of the molecular mechanisms controlling osteogenic differentiation of periodontal ligament cell progenitors remains poor.

In this study, we investigated the effects of hypoxia on HPDLSC proliferation and osteogenic differentiation.

\section{MATERIAL AND METHODS}

\section{Hypoxia}

Hypoxia was achieved using a sealed jar (Oxoid Ltd.; Basingstoke, UK) containing an oxygen chelator (AnaeroGen; Oxoid, Ltd.; Hampshire, UK). In the experimental group, $\mathrm{pO}_{2}$ was adjusted to $2 \%$ and measured using an oxygen electrode placed directly in the cell culture medium ( $\mathrm{pH}$ 7.2) and using an Oxylab $\mathrm{pO}_{2}{ }^{\mathrm{TM}}$ system (Oxford Optronix; Oxford, UK). The hypoxic system remained closed during the experiment. In the control group, $\mathrm{pO}_{2}$ was maintained at $21 \%$ throughout the experiment.

\section{Cell culture}

Teeth were obtained with the permission of patients for orthodontic reasons. Patients provided written informed consent to publish the case details. Teeth were obtained from 3 donors who were of Han Chinese nationality and 18-20 years old. Pieces of periodontal ligament were obtained exclusively from the middle of tooth roots to exclude intermixture of gingiva and dental pulp. Ligament samples were cultured in a-minimum essential media (a-MEM) containing $10 \%$ fetal bovine serum (FBS) and supplemented with antibiotics on a $35-\mathrm{mm}$ primary culture dish (Falcon Becton Dickinson; Franklin Lakes, NJ, USA). HPDLSCs used in these experiments underwent 3 passages.

\section{Surface markers of HPDLSCs analyzed by flow cytometry}

Logarithmic growth phase HPDLSCs (passage 1 or 2) were digested with $0.25 \%$ trypsin for $2 \mathrm{~min}$, centrifuged for $5 \mathrm{~min}$ at $1000 \mathrm{rpm}$, and the supernatant was discarded. Cell density was adjusted to $1 \times 10^{6}$ cells $/ \mathrm{mL}$, washed twice with phosphate-buffered saline (PBS), and resuspended in $0.02 \mathrm{~mL} \mathrm{FCM}$ buffer (containing $20 \mathrm{~g} / \mathrm{L}$ bovine serum albumin and $0.1 \mathrm{~g} / \mathrm{L}$ sodium azide). The PE biomarkers STRO-1 and CD146 fluorescein isothiocyanate-antibodies were added and the sample was incubated in the dark at $4^{\circ} \mathrm{C}$ for $20 \mathrm{~min}$, resuspended in 0.2 $\mathrm{mL}$ PBS, and examined using flow cytometry.

\section{Induction of osteogenic and adipogenic differentiation}

HPDLSCs (passage 1 or 2) were cultured in osteogenic or adipogenic medium for 7 days. Osteogenic media is composed of a-MEM containing 10\% FBS, 10-7 M dexamethasone (Sigma; St. Louis, MO, USA), $0.15 \mathrm{mM}$ ascorbate-2-phosphate (Sigma), and $2 \mathrm{mM}$ b-glycerophosphate (Sigma). Adipogenic medium is composed of a-MEM containing 10\% FBS, $1 \mathrm{mM}$ dexamethasone, $0.5 \mathrm{mM}$ 3-isobutyl-1-methylxanthine, $100 \mathrm{mg} / \mathrm{L}$ indomethacin, and $10 \mathrm{mg} / \mathrm{L}$ 
insulin. After 21 days of culture, calcium deposition was assayed using Alizarin Red staining for calcium nodules. After 7 days of culture, lipid deposition was assayed using the Oil Red O staining lipid droplet test.

\section{Growth potential}

To assess growth potential, P1 and P2 HPDLSCs were counted and seeded on four $100-\mathrm{mm}$ Petri dishes at $1.5 \times 10^{5}$ cells/dish. Two dishes were placed in normoxic conditions and two in hypoxic conditions. On day 7, the cells were harvested, re-suspended in $10 \mathrm{~mL}$ culture medium, and a $0.5-\mathrm{mL}$ sample was used to determine cell concentration.

\section{Semi-quantitative reverse transcription-polymerase chain reaction (RT-PCR)}

HPDLSCs (passage 3) were seeded at $5000 \mathrm{cells} / \mathrm{cm}^{2}$ and allowed to adhere to the plate overnight. After exposure either to hypoxic or control conditions for 7 days, the cell culture supernatant medium was replaced with osteogenic medium. HPDLSCs were cultured under control conditions for 7 days. Semi-quantitative RT-PCR was performed to determine the transcription levels of 3 typical osteogenic markers, osteopontin, osteocalcin, and corebinding factor a $1(C b f a-1) /$ runt-related transcription factor 2 (Runx2).

Total RNA was prepared using TRIzol reagent (Invitrogen; Carlsbad, CA, USA) according to the manufacturer protocol. The SuperScript II First-Strand Synthesis System for RT-PCR was used to synthesize cDNA. Relative mRNA expression levels of osteopontin, osteocalcin, and $C b f a-1 / R u n x 2$ were determined by PCR using glyceraldehyde 3-phosphate dehydrogenase as a positive control. The primers for these genes are shown in Table 1. PCR was performed using Taq DNA polymerase (New England Biolabs; Ipswich, MA, USA) under the following amplification conditions: denaturation at $94^{\circ} \mathrm{C}$ for $2 \mathrm{~min}$, followed by 30 cycles at $94^{\circ} \mathrm{C}$ for $30 \mathrm{~s}, 56^{\circ} \mathrm{C}$ for $40 \mathrm{~s}, 72^{\circ} \mathrm{C}$ for $50 \mathrm{~s}$, and extension at $72^{\circ} \mathrm{C}$ for $5 \mathrm{~min}$. PCR products were subjected to $1.5 \%$ agarose gel electrophoresis. The semi-quantitative method was used to evaluate relative gene transcription levels. The Image $J$ software (National Institutes of Health; Bethesda, MD, USA) was used to determine the gray intensity values of the osteopontin, osteocalcin, and $C b f a-1 / R u n x 2$ bands, which were corrected based on glyceraldehyde 3-phosphate dehydrogenase gene expression of the same sample.

Table 1. PCR primer sequence and size.
\begin{tabular}{lllc}
\hline Gene name & Forward (5'-3') & Reverse $\left(5^{\prime}-3^{\prime}\right)$ & Product size \\
\hline GAPDH & ggagcgagatccetccaaaat & ggctgttgtcatacttctcatgg & $197 \mathrm{bp}$ \\
Osteopontin & tgaaacgagtcagctggatg & tgaaattcatggctgtggaa & $162 \mathrm{bp}$ \\
Osteocalcin & ccccgettcctctttagact & agggtgagccacaatcagac & $163 \mathrm{bp}$ \\
Cbfa-1/Runx2 & ttgcactgggtcatgtgtt & tggctgcattgaaaagactg & $156 \mathrm{bp}$ \\
\hline
\end{tabular}

\section{In vivo experiment}

After 7 days of exposure to hypoxia and normal conditions, each cell group was transplanted subcutaneously into the back of immunocompromised mice to determine differences in osteogenic function in vivo. Cyclosporine A was used at $15 \mathrm{mg} / \mathrm{kg}$ body weight, administered $24 \mathrm{~h}$ before transplantation and then daily, reducing the dose to $6 \mathrm{mg} / \mathrm{kg}$ over a 2 -week 
period. This dose was administered until animals were sacrificed 12 weeks after transplantation. Hematoxylin and eosin staining was conducted according to the protocol described by the Rosen lab (http://www.bcm.edu/rosenlab).

\section{Ethics statement}

The study was approved by the institutional ethics board of the Hospital of Stomatology, Guangzhou Medical University, including the procedures involving human dental tissue and mouse experiments. All efforts were made to minimize the suffering of mice. All mice were sacrificed by cervical dislocation and decapitations and all surgeries were performed under anesthesia with $2 \mathrm{mg} / \mathrm{kg}$ urethane. Written informed consent forms were signed by the patients or parents before the investigation began.

\section{Statistical analysis}

Data are reported as means \pm standard deviation. Statistical analysis was performed using 1-way or 2-way analysis of variance followed by the Fisher post-hoc test. The results were considered to be significant at a probability level of $\mathrm{P}<0.05$.

\section{RESULTS}

\section{Culture and identification of HPDLSCs}

We isolated, identified, and assessed the characteristics of HPDLSCs using in vitro methods. Periodontal ligament cells were obtained from teeth extracted for orthodontic purposes and used to isolate HPDLSCs using a limiting dilution assay, which is an effective method for isolating HPDLSCs. Single cell-derived colonies demonstrate similar properties to stem cells in vitro. HPDLSCs were cultured and expanded in a-MEM supplemented with $10 \%$ FBS. A colony-forming assay, immunohistochemistry, flow cytometry, and osteogenic and adipogenic induction were also used to identify HPDLSCs. The cells obtained showed high colony-forming efficiency (Figure 1A, white arrow). Flow cytometry revealed that the percent of STRO-1 positive HPDLSCs was $5.36 \pm 2.34 \%(\mathrm{P} 1)$ and $4.43 \pm 1.46 \%(\mathrm{P} 2)$, and the percent of CD146-positive HPDLSCs was $30.27 \pm 3.52 \%(\mathrm{P} 1)$ and $26.45 \pm 3.59 \%(\mathrm{P} 2)$
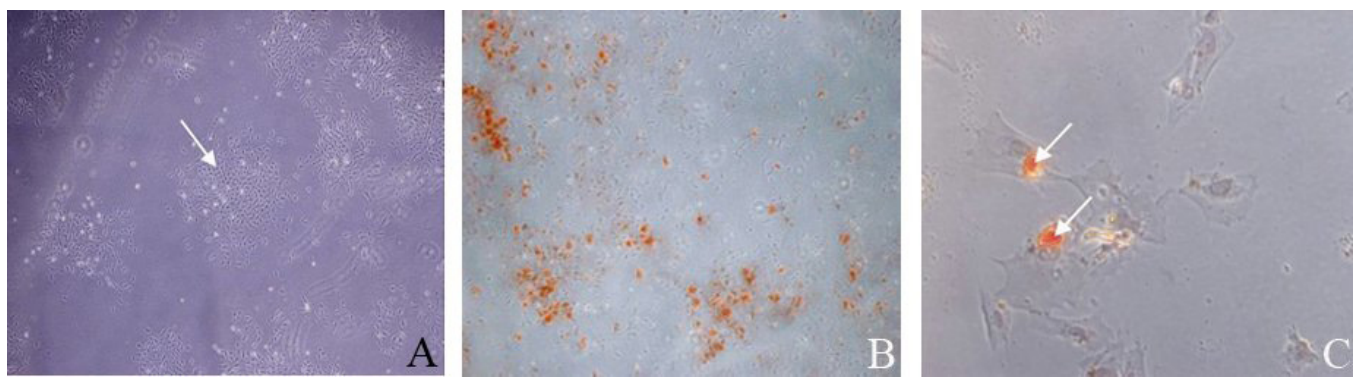

Figure 1. A. Colony characteristics of periodontal ligament stem cells. B. Alizarin Red-positive nodules formed in the human periodontal ligament stem cell (HPDLSC) cultures after osteogenic induction. C. Oil Red O-positive nodules formed in the HPDLSC cultures after adipogenic induction. 
antibodies. Under specific conditions, the cells could differentiate into the osteoblast and adipocyte lineage in vitro. Small, round Alizarin Red-positive nodules formed in the HPDLSC cultures after osteogenic induction (Figure 1B), indicating calcium accumulation in vitro. Small, round Oil Red O-positive nodules formed in the HPDLSC cultures after adipogenic induction (Figure 1C), indicating lipid droplet accumulation in vitro.

\section{In vitro investigation}

A traditional method was utilized to create the hypoxic conditions. To evaluate the validity of the model for hypoxia used in this study, $\mathrm{pO}_{2}$ levels were monitored in a sealed jar for 7 days without exposure to atmospheric oxygen. Lower oxygen concentrations can increase the proliferation effect on multiple stem cell types. However, the effects of prolonged hypoxia on HPDLSC survival were unclear. In this study, we investigated the effects of hypoxia on cell proliferation of HPDLSCs. HPDLSCs cultured under hypoxic conditions showed higher cell numbers than under normal oxygen conditions after 7 days of passages 1 and 2 (Figure 2). The effects on HPDLSC osteogenic potential in vitro were also assessed. After 7 days of exposure to hypoxic or control conditions, HPDLSCs were transferred to osteogenic medium and osteogenic differentiation was assessed using RT-PCR to detect the expression of several osteogenic markers. Osteopontin expression increased after exposure to hypoxic conditions for 7 days. Cbfa-1/Runx 2 and osteocalcin expression levels were also increased after 7 days of osteogenic culture following exposure to hypoxic conditions (Figure 3) $(\mathrm{P}<0.05)$.

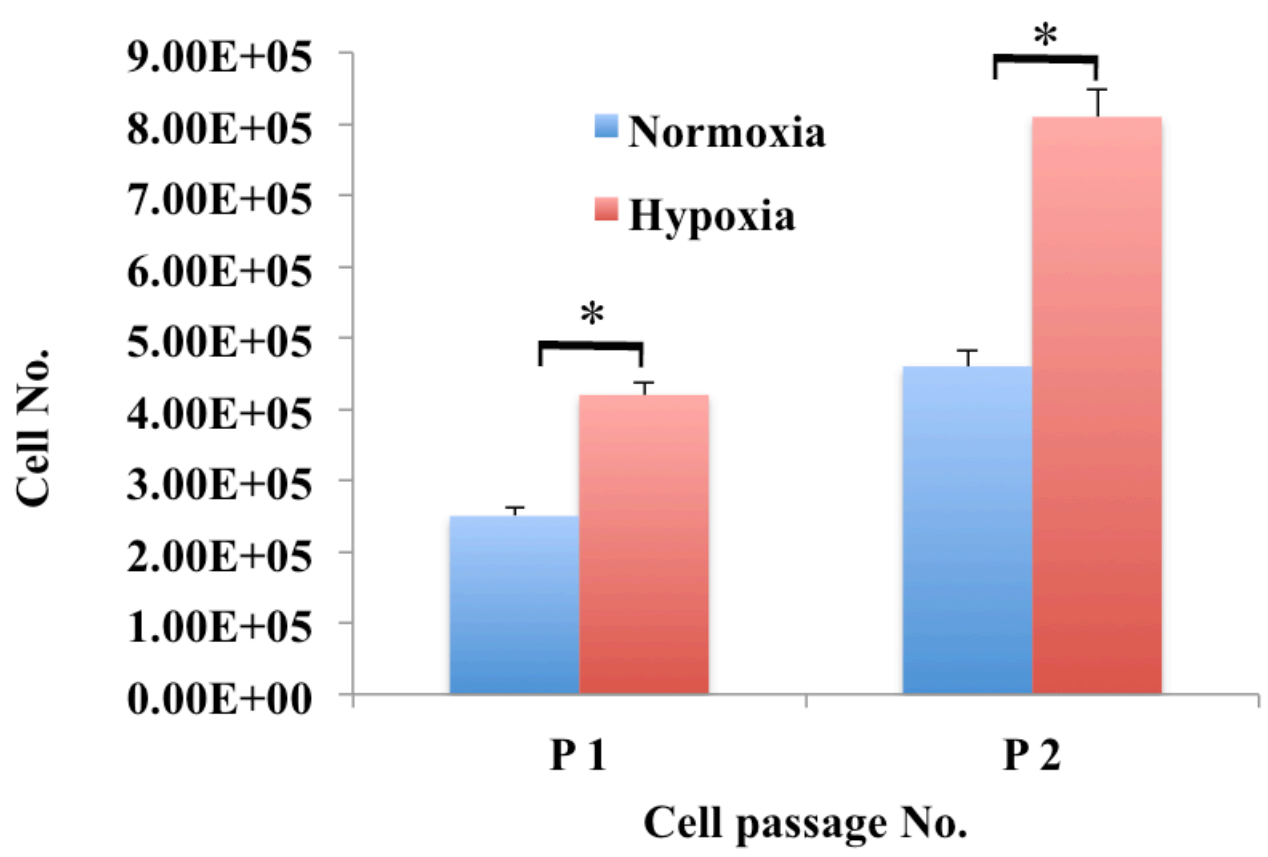

Figure 2. Proloferation of periodontal ligament stem cells under hypoxia and normoxia situations. 


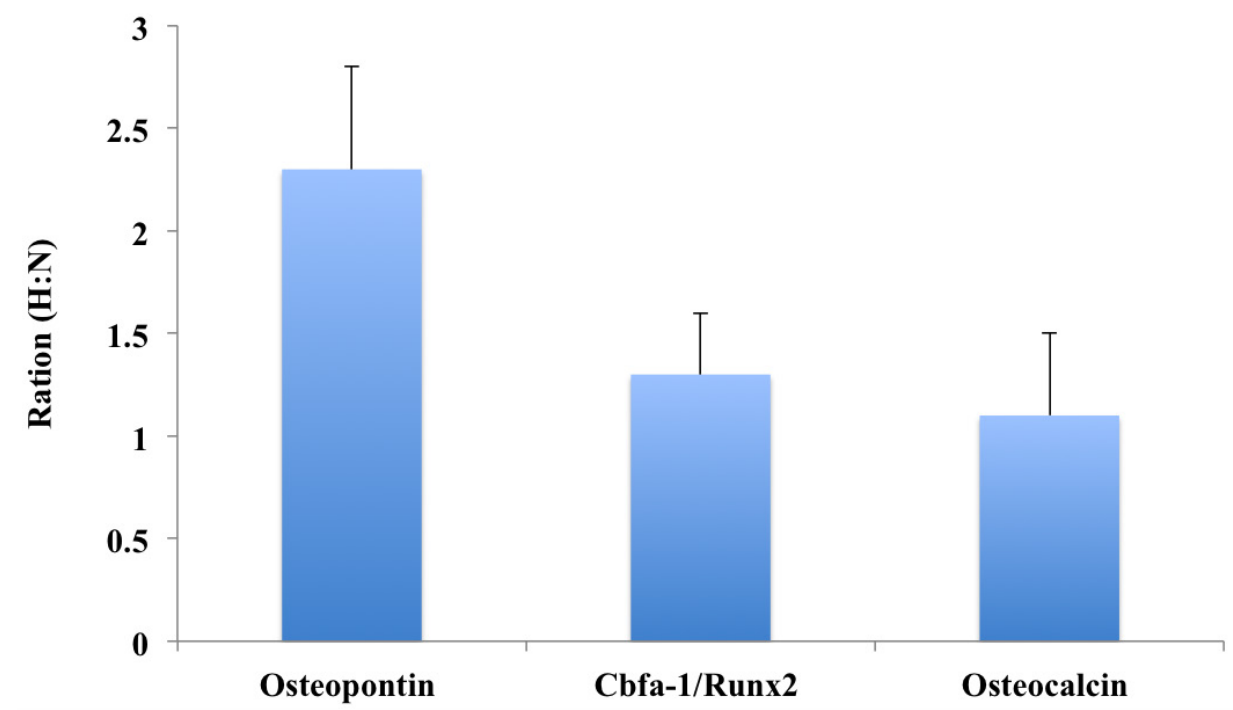

Figure 3. Different expression of Cbfa-1/Runx2 and osteocalcin levels after exposure to hypoxic conditions.

\section{In vivo investigation}

Twelve weeks after transplantation, both HPDLSCs and hypoxia-treated HPDLSCs showed a large amount of newly formed bone, while most of the hydroxyapetite-like particles had been resorbed. Many osteocytes trapped in osteocytic lacunae and fiber tissues were also poorly represented. In addition, well-formed granulomas and an acute inflammation response were absent (Figure 4A). Histomorphometric data showed that the mean width of trabecular, number of osteocytes, number of osteoclasts, and quantity of cancellous bone in the defect area were significantly upregulated in the hypoxia-treated HPDLSC group compared with the HPDLSCs group $(\mathrm{P}<0.05)$ (Figure 4B). Immunohistochemical staining with anti-human mitochondria antibody showed that HPDLSCs and hypoxia-treated HPDLSCs were positive and generated bone-like structures from osteoblast-like cells (Figure 5A). The mean optical density of hypoxia-treated HPDLSCs was significantly higher than that of the control group $(\mathrm{P}<0.05)$ (Figure 5B).
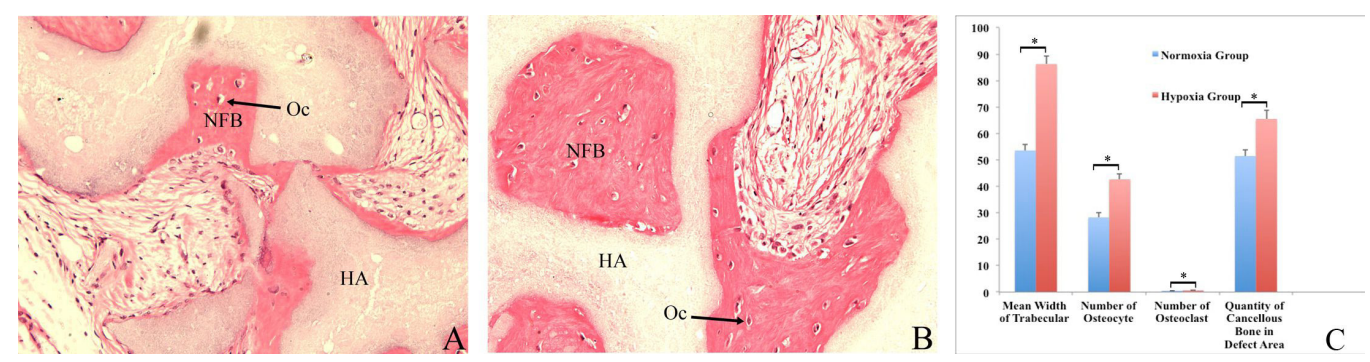

Figure 4. A. Absence of well-formed granulomas and an acute inflammation response. B. Histomorphometric differences between human periodontal ligament stem cell (HPDLSC) group and hypoxia-treated HPDLSC group. C. Different osteogenic ability in HPDLSC group and hypoxia-treated HPDLSC group. 

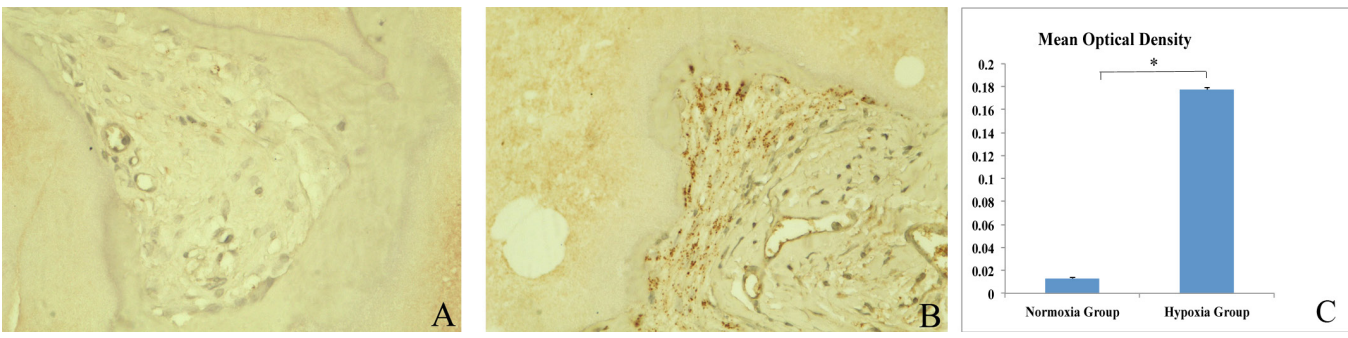

Figure 5. Changing expression of immunohistochemical staining with anti-human mitochondrium antibody in human periodontal ligament stem cell (HPDLSC) group and hypoxia-treated HPDLSC group.

\section{DISCUSSION}

HPDLSCs are stem cells present in the periodontal ligament of teeth. They are involved in adult regeneration of the periodontal tissue, including alveolar bone and the periodontal membrane (Robins et al., 2005; Ren et al., 2006). The proportion of cells that possess stem cell properties in human periodontal ligament cells is not well known, although it was first reported by Seo et al. (2004). Previous reports showed that HPDLSCs were clonogenic and positive for typical markers of STRO-1 and CD146 (Wang et al., 2005). The cells had the capacity to undergo osteogenic and adipogenic differentiation in vitro. Stem cells have been used for regenerative therapies in various fields (Lennon et al., 2001; Annabi et al., 2003). Adult stem cells must persist throughout life to ensure continuous replenishment of dead or damaged cells in various tissues of the body. While numerous studies have already identified some of the factors and mechanisms regulating the long-term function and survival of stem cells (D'Ippolito et al., 2004), these processes are not well understood. A growing body of evidence suggests that various types of stem cells exist in a hypoxic microenvironment, which may be conducive to stem cell longevity.

Osteogenic differentiation is an important cell characteristic of HPDLSCs, both in biological research and clinical trials (Sekiya et al., 2002; Grayson et al., 2004). HPDLSCs have the innate nature of osteogenesis; numerous biological factors have been utilized to promote the osteogenic process, and positive results have been reported. However, the defined molecular factors that specifically induce the osteoblastic phenotype have not been thoroughly explored in periodontal ligament cells (Serakinci et al., 2004). Biologically and anatomically, the periodontal ligament is a non-mineralized connective tissue surrounding the tooth and exhibits osteoblast-like features, such as high alkaline phosphatase activity and expression of osteogenic markers (Trosko et al., 2004; Wong et al., 2004). In addition, RNA expression of bone sialoprotein and osteopontin was detectable in cells obtained from freshly isolated periodontal ligament tissue, with much lower levels found in cultured periodontal ligament cells (Cheng et al., 2004).

Recently, hypoxia has been widely explored to determine the effects on stem cell differentiation (Zhang et al., 2002; Klepsch et al., 2013). A previous study reported varying results for this analysis (Shav-Tal et al., 2001). Some studies reported that hypoxia provides favorable culture conditions for promoting proliferation as well as osteogenesis of mesenchymal stem cells through differential growth factor production (Wiblin et al., 2005). Changes in oxygen concentrations affect many of the innate characteristics of stem and progenitor cells. Human mesenchymal stem cells were maintained under hypoxic atmospheres $\left(2 \% \mathrm{O}_{2}\right)$ for up 
to 7 in vitro passages. Oxygen concentrations affected various aspects of stem-cell physiology, including growth and in vitro development, and may be a critical parameter during expansion and differentiation. Studies showed that hypoxia and the hypoxia-mimicking agent $\mathrm{CoCl}_{2}$ may trigger the differentiation of acute promyelocytic leukemia cells, and that variations in oxygen concentration modified the self-renewal and cycling of hematopoietic stem cells (Lee et al., 2007). It has also been reported that $1 \%$ hypoxia modified the proliferation and differentiation of $\mathrm{CD} 34^{+}$chronic myelogenous leukemia cells. Furthermore, the proportion of osteochondrogenesis in Iscove's modified Dulbecco's medium containing 10\% FBS under hypoxic conditions $\left(5 \% \mathrm{O}_{2}\right.$ concentration) was higher than that under normoxic conditions in vitro and in vivo.

However, few studies have examined the effects of hypoxia on HPDLSCs. Low oxygen tension is a potent differentiation inducer of numerous cell types and an effective stimulus of gene expression (Rosafio and Pellerin, 2013). Thus, we evaluated the effects of reduced oxygen tension on HPDLSCs proliferation and the influence on osteogenic capability. After 7 days exposure to hypoxic and control conditions, HPDLSCs were transferred to osteogenic medium and osteogenic differentiation capacity was evaluated by RT-PCR to detect the expression of Oct-4 and several osteogenic markers, including Cbfa-1/Runx2, osteopontin, and osteocalcin. Alkaline phosphatase and osteopontin expression increased after exposure of HPDLSCs to hypoxic conditions. Expression levels of Cbfa-1/Runx2 and osteocalcin were also upregulated after 7 days of osteogenic induction. The mechanisms underlying HPDLSC death upon oxygen deprivation were not well understood. Our results and those of previous studies suggest that hypoxia leads only to moderate cell death and that the surviving HPDLSCs are still able to proliferate. However, the ultimate bone forming ability of engineered constructs relies on the survival of "functional" HPDLSCs. Our results showed that slight upregulation of $C b f a-1 / R u n x 2$ expression occurs after exposure to hypoxia. Additionally, Oct4, a marker of primitive stem cells, was also upregulated. Oct4 represents the most important marker of pluripotent stem cells, such as embryonic stem cells and multipotent adult progenitor cells (Shi and Jin, 2010). HPDLSCs in culture expressed Oct4, indicating that at least a subpopulation of cells in HPDLSCs may be pluripotent-like embryonic stem cells. Therefore, it is important to identify Oct4-positive cells in stem cell lines and other origins to determine their plasticity. The upregulation or downregulation of these genes may represent the differentiation potential of HPDLSCs. We found that $2 \%$ hypoxia triggered differentiation of Oct4-positive cells within 7 days under hypoxia conditions. Regarding osteogenesis in vivo, hypoxic conditions clearly enhanced new bone-like tissue formation compared to cells cultured under normal conditions. Previous studies conducted using other cell types reported that their osteogenic differentiation is impaired by temporary exposure to hypoxia (decreased alkaline phosphatase activity, collagen type I, osteocalcin, and Cbfa-1/Runx2 expression). In contrast, Zhu et al. (2013) reported that exposure of human mesenchymal stem cells to hypoxic $\left(2 \% \mathrm{O}_{2}\right)$ conditions did not affect their terminal differentiation. Our results suggest that HPDLSCs can tolerate hypoxia for a period of time (at least 7 days) without losing their osteogenic potential. In contrast, alkaline phosphatase, Cbfa-1/Runx2, osteopontin, osteocalcin, and Oct-4 expression by HPDLSCs were permanently increased. Thus, further studies should be conducted to determine the in vitro HPDLSC culture conditions that are most appropriate for preserving the osteogenic potential after in vivo implantation.

Our results demonstrate that hypoxia accelerates HPDLSC proliferation and differentiation in the osteogenic direction. Future studies examining the mechanism underlying these 
effects may increase the understanding of the relationship between hypoxia and stem cell maintenance.

\section{Conflicts of interest}

The authors declare that there is no conflict of interest.

\section{ACKNOWLEDGMENTS} (\#2013294).

Research supported by the medical research foundation of Guangdong province

\section{REFERENCES}

Annabi B, Lee YT, Turcotte S, Naud E, et al. (2003). Hypoxia promotes murine bone-marrow-derived stromal cell migration and tube formation. Stem Cells 21: 337-347.

Baksh D, Davies JE and Zandstra PW (2003). Adult human bone marrow-derived mesenchymal progenitor cells are capable of adhesion-independent survival and expansion. Exp. Hematol. 31: 723-732.

Bruder SP, Jaiswal N and Haynesworth SE (1997). Growth kinetics, self-renewal, and the osteogenic potential of purified human mesenchymal stem cells during extensive subcultivation and following cryopreservation. J. Cell. Biochem. 64: 278-294.

Cheng A, Tang H, Cai J, Zhu M, et al. (2004). Gap junctional communication is required to maintain mouse cortical neural progenitor cells in a proliferative state. Dev. Biol. 272: 203-216.

D’Ippolito G, Diabira S, Howard GA, Menei P, et al. (2004). Marrow-isolated adult multilineage inducible (MIAMI) cells, a unique population of postnatal young and old human cells with extensive expansion and differentiation potential. J. Cell Sci. 117: 2971-2981.

Ezashi T, Das P and Roberts RM (2005). Low $\mathrm{O}_{2}$ tensions and the prevention of differentiation of hES cells. Proc. Natl. Acad. Sci. U. S. A. 102: 4783-4788.

Grayson WL, Ma T and Bunnell B (2004). Human mesenchymal stem cells tissue development in 3D PET matrices. Biotechnol. Prog. 20: 905-912.

Grayson WL, Zhao F, Izadpanah R, Bunnell B, et al. (2006). Effects of hypoxia on human mesenchymal stem cell expansion and plasticity in 3D constructs. J. Cell. Physiol. 207: 331-339.

Ivanović Z, Bartolozzi B, Bernabei PA, Cipolleschi MG, et al. (2000a) Incubation of murine bone marrow cells in hypoxia ensures the maintenance of marrow-repopulating ability together with the expansion of committed progenitors. $\mathrm{Br}$. J. Haematol. 108: 424-429.

Ivanović Z, Dello Sbarba P, Trimoreau F, Faucher JL, et al. (2000a). Primitive human HPCs are better maintained and expanded in vitro at 1 percent oxygen than at 20 percent. Transfusion 40: 1482-1488.

Klepsch S, Jamnig A, Trimmel D, Schimke M, et al. (2013). Isolation of mesenchymal stem cells from human bone and long-term cultivation under physiologic oxygen conditions. Methods Mol. Biol. 976: 99-109.

Lee JH and Kemp DM (2006). Human adipose-derived stem cells display myogenic potential and perturbed function in hypoxic conditions. Biochem. Biophys. Res. Commun. 341: 882-888.

Lee H, Bien CM, Hughes AL, Espenshade PJ, et al. (2007). Cobalt chloride, a hypoxia-mimicking agent, targets sterol synthesis in the pathogenic fungus Cryptococcus neoformans. Mol. Microbiol. 65: 1018-1033.

Lennon DP, Edmison JM and Caplan AI. (2001). Cultivation of rat marrow-derived mesenchymal stem cells in reduced oxygen tension: effects on in vitro and in vivo osteochondrogenesis. J. Cell Physiol. 187: 345-355.

Lombardo L, Bragazzi R, Perissinotto C, Mirabella D, et al. (2013). Cone-beam computed tomography evaluation of periodontal and bone support loss in extraction cases. Prog. Orthod. 14: 29.

Malladi P, Xu Y, Chiou M, Giaccia AJ, et al. (2006). Effect of reduced oxygen tension on chondrogenesis and osteogenesis in adipose-derived mesenchymal cells. Am. J. Physiol. Cell Physiol. 290: C1139-C1145.

Pittenger MF, Mackay AM, Beck SC, Jaiswal RK, et al. (1999). Multilineage potential of adult human mesenchymal stem cells. Science 284: 143-147.

Ren H, Cao Y, Zhao Q, Li J, et al. (2006). Proliferation and differentiation of bone marrow stromal cells under hypoxic conditions. Biochem. Biophys. Res. Commun. 347: 12-21. 
Robins JC, Akeno N, Mukherjee A, Dalal RR, et al. (2005). Hypoxia induces chondrocyte-specific gene expression in mesenchymal cells in association with transcriptional activation of Sox9. Bone 37: 313-322.

Rosafio K and Pellerin L (2013). Oxygen tension controls the expression of the monocarboxylate transporter MCT4 in cultured mouse cortical astrocytes via a hypoxia-inducible factor- $1 \alpha$-mediated transcriptional regulation. Glia 62 : 477-490.

Sekiya I, Larson BL, Smith JR, Pochampally R, et al. (2002). Expansion of human adult stem cells from bone marrow stroma: conditions that maximize the yields of early progenitors and evaluate their quality. Stem Cells 20: 530-541.

Seo BM, Miura M, Gronthos S, Bartold PM, et al. (2004). Investigation of multipotent postnatal stem cells from human periodontal ligament. Lancet 364: 149-155.

Serakinci N, Guldberg P, Burns JS, Abdallah B, et al. (2004). Adult human mesenchymal stem cell as a target for neoplastic transformation. Oncogene 23: 5095-5098.

Shav-Tal Y, Lee BC, Bar-Haim S, Schori H, et al. (2001). Reorganization of nuclear factors during myeloid differentiation. J. Cell. Biochem. 81: 379-392.

Shi G and Jin Y (2010). Role of Oct4 in maintaining and regaining stem cell pluripotency. Stem Cell Res. Ther. 1: 39.

Trosko JE, Chang CC, Upham BL and Tai MH (2004). Ignored hallmarks of carcinogenesis: stem cells and cell-cell communication. Ann. N Y Acad. Sci. 1028: 192-201.

Wang DW, Fermor B, Gimble JM, Awad HA, et al. (2005). Influence of oxygen on the proliferation and metabolism of adipose derived adult stem cells. J. Cell Physiol. 204: 184-191.

Wiblin AE, Cui W, Clark AJ and Bickmore WA (2005). Distinctive nuclear organisation of centromeres and regions involved in pluripotency in human embryonic stem cells. J. Cell Sci. 118: 3861-3868.

Wong RC, Pébay A, Nguyen LT, Koh KL, et al. (2004). Presence of functional gap junctions in human embryonic stem cells. Stem Cells 22: 883-889.

Zhang W, Green C and Stott NS (2002). Bone morphogenetic protein-2 modulation of chondrogenic differentiation in vitro involves gap junction-mediated intercellular communication. J. Cell. Physiol. 193: 233-243.

Zhu LL, Wu LY, Yew DT and Fan M (2005). Effects of hypoxia on the proliferation and differentiation of NSCs. Mol. Neurobiol. 31: 231-242.

Zhu M, Feng Q and Bian L (2013). Differential effect of hypoxia on human mesenchymal stem cell chondrogenesis and hypertrophy in hyaluronic acid hydrogels. Acta Biomater. 10: 1333-1340. 